\title{
Suspended Single-Wall Carbon Nanotubes as a Sensor of Molecular Adsorption
}

\author{
Yoshikazu Homma*, Tateki Hanashima and Shohei Chiashi ${ }^{1}$ \\ Department of Physics, Tokyo University of Science, Shinjuku-ku, Tokyo 162-8601, Japan \\ ${ }^{1}$ Department of Mechanical Engineering, University of Tokyo, \\ Bunkyo-ku, Tokyo 113-8656, Japan
}

(Received July 27, 2009; accepted July 30, 2009)

Key words: carbon nanotubes, photoluminescence, environmental effect, adsorption, desorption

All the carbon atoms of a single-wall carbon nanotube (SWNT) are in the surface layer. Thus, SWNTs suffer from strong interaction with the surrounding environment. When an SWNT is suspended between mesa structures, the interactions with the substrate and other nanotubes are minimized, and the nanotube is directly exposed to ambient gas. Semiconducting SWNTs suspended in space exhibit intense photoluminescence, and their optical transition energy depends on the state of molecules absorbed onto the SWNT surface. Therefore, gas molecule adsorption/desorption can be probed by photoluminescence analysis.

\section{Introduction}

Single-wall carbon nanotubes (SWNTs) exhibit chemical stability and mechanical strength, owing to their stable carbon-carbon bonds, and features specific to the onedimensional structure, which makes them attractive as building blocks for nanoelectronic and nanophotonic devices. ${ }^{(1)}$ Semiconducting SWNTs have a direct band gap, and thus light emission from electron-hole recombination should occur. However, SWNTs lying on a substrate surface, as well as SWNT bundles, show almost no luminescence. This means that an SWNT, whose constituent carbon atoms are all in the surface layer, is strongly affected by the surroundings, and the electronic transitions are modified. By contrast, individual SWNTs dispersed in solution ${ }^{(2)}$ or suspended between mesa structures, emit a bright band-gap photoluminescence (PL). ${ }^{(3)}$ Thus, the isolation of SWNTs by minimizing their interaction is a key to extracting the intrinsic optical properties of nanotubes. Since suspended SWNTs do not come in contact with substrates or any surrounding medium and exhibit intense and sharp PL peaks ${ }^{(3)}$ suspended SWNTs are ideal for PL measurement and the determination of optical properties.

Lefebvre et al. first observed the effects of environment on PL excitation and emission energy by comparing the PLs of seven chiralities between air-suspended and micelle

"Corresponding author: e-mail: homma@rs.kagu.tus.ac.jp 
(sodium dodecyl sulfate: SDS)-wrapped SWNTs. ${ }^{(4)}$ They found that the optical transition energies of SDS-wrapped SWNTs redshift by an average of $28 \mathrm{meV}$ for emission and by an average of $16 \mathrm{meV}$ for excitation compared with those of air-suspended SWNTs. The origin of the optical transition energy shift is dielectric screening by the nanotube' s surrounding environment. Dielectric screening has two opposite effects: a decrease in Coulomb self-energy (electron-electron interaction) and a decrease in exciton binding energy. Since the self-energy is larger in magnitude than the exciton binding energy, the overall energy decreases, i.e., a redshift is predicted theoretically. ${ }^{(5)}$ Experimentally, Ohno et al. examined the dependence of excitonic transition energy on the environmental dielectric constant by immersing suspended SWNTs in various organic solvents. ${ }^{(6)}$ For all organic solvents, both the emission and excitation energies of nine chiralities redshifted relative to those of air-suspended SWNTs. These results are in good agreement with the theoretical calculations by Miyauchi et al. ${ }^{(7)}$

In gas ambient, the PL emission peaks of suspended SWNTs rapidly change at approximately $40^{\circ} \mathrm{C} .{ }^{(8)}$ Transition temperature shows a clear diameter dependence: transition temperature increases with emission wavelength, which is proportional to tube diameter. This phenomenon is interpreted in terms of molecule adsorption/desorption. Here, we focus on the molecule-induced transitions of PL energies. This is of interest for the application of molecular sensing as well as for the basic study of molecule adsorption/desorption on a nanosurface.

\section{Photoluminescence of Suspended SWNTs}

The preparation procedures for suspended SWNTs are described elsewhere. ${ }^{(9)}$ A scanning electron microscopy (SEM) image of an SWNT suspended between silica pillars is shown in Fig. 1. Since suspended SWNTs tend to form bundles, a short growth time (5 min) in chemical vapor deposition was used to prevent bundle formation. A PL excitation (PLE) map is a two-dimensional plot of PL excitation and emission spectra (Fig. 2). The peak in the PLE plot represents the optical transition energies between 1D singular peaks of semiconducting SWNT, and can be used for determining the chiral indices of SWNTs. ${ }^{(10)}$ In Fig. 2, a single PL peak is seen, indicating the existence of

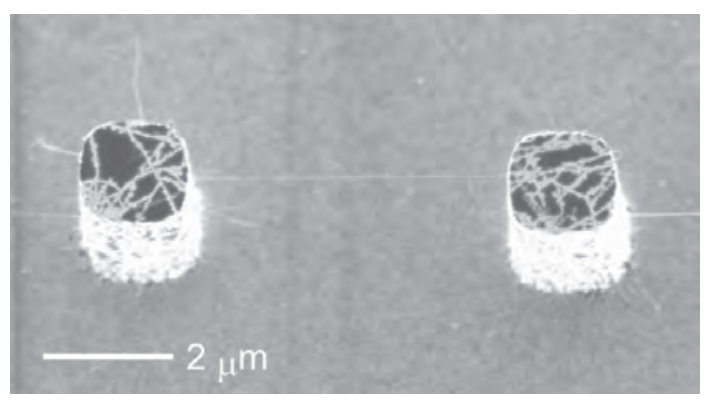

Fig. 1. SEM image of a SWNT suspended between silica pillars. 


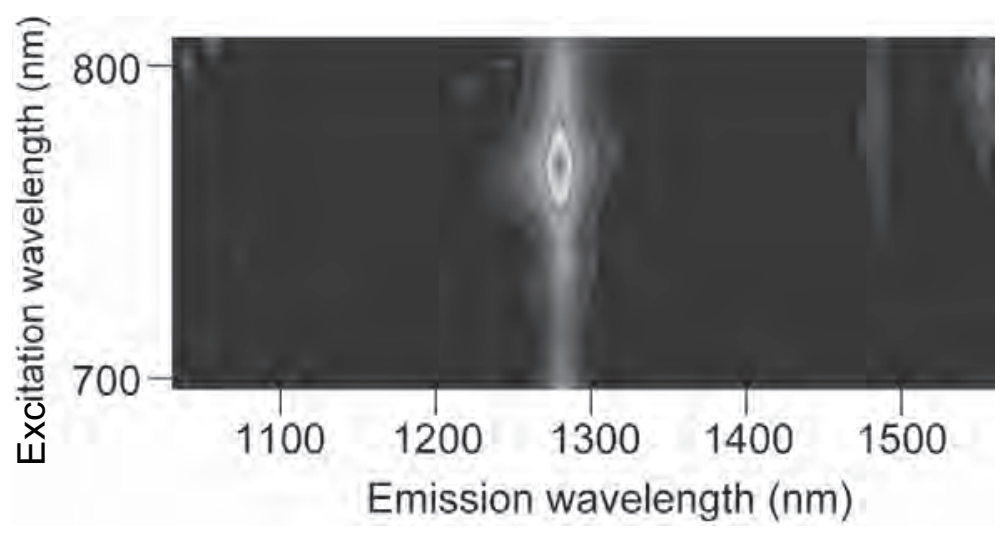

Fig. 2. PLE map for an SWNT suspended between silica pillars.

a single SWNT between a pair of pillars, and the peak corresponds to an excitation of $1.6 \mathrm{eV}$ and an emission of $0.96 \mathrm{eV}$. By taking into account the energy shift between suspended SWNTs and micelle-wrapped SWNTs, ${ }^{(11)}$ the chiral index of the SWNT is identified as $(9,7)$.

In PLE mapping, the excitation energy range is determined by the light source. For the imaging of a single SWNT, a light power range of $1-5 \mathrm{~mW}$ is necessary. Thus, a Ti: sapphire laser $(700-1,050 \mathrm{~nm})$ is commonly used. The wavelength range corresponds to the excitation energy between second 1D singularities of nanotube electronic structures with a diameter of $1 \mathrm{~nm}$ or larger. For the excitation of nanotubes with smaller diameters, a wavelength range of $500-700 \mathrm{~nm}$ is necessary. The emission wavelength is in the near-infrared regime, $>800 \mathrm{~nm}$. An InGaAs photodiode is typically sensitive to the 1,000-1,600 nm wavelength range, which corresponds to emission for nanotubes with diameters ranging from 0.9 to $1.3 \mathrm{~nm}$. A diode array with 512 or 1,024 channels is used for rapid spectroscopic imaging. ${ }^{(3)}$

Because of the one-dimensionality of nanotubes, photoexcitation and photoemission show strong polarization dependences. Both excitation and emission have a maximum for excitation laser polarization parallel to the nanotube axis. A square-cosine dependence has been reported. ${ }^{(12)}$ This is due to the polarization-dependent selection rule of optical transitions between 1D singular peaks. For polarization parallel to the tube axis, optical absorption between subbands with the same angular momentum are allowed. When used, transverse-light polarization induces a transverse polarization in the nanotube, which has a shielding effect on electric field. ${ }^{(13)}$ Thus, the absorption and emission intensities are always weak in the perpendicular direction.

The actual scheme of optical transitions in nanotubes is different from the simple single-particle picture. Since excited electrons and holes are confined in a onedimensional nanotube, they interact strongly, forming hydrogen-atom-like binding states, or excitons. ${ }^{(14)}$ Thus, the transition energies are different from those expected from the 
simple single-particle picture. In the excitonic excitation picture, the transition energy is the sum of the band gap and exciton binding energy (attractive interaction between an electron and a hole), which are affected by dielectric screening by the nanotube's surrounding environment.

\section{Gas Adsorption Effect}

At temperatures higher than room temperature, the PL emission peaks of suspended SWNTs rapidly change at approximately $40^{\circ} \mathrm{C}$ in gas ambient and vacuum. ${ }^{(8)}$ Transition temperature shows a clear diameter dependence: transition temperature increases with emission wavelength, which is proportional to tube diameter. Transition temperature also depends on the ambient gas species. It is higher for air and nitrogen than for helium. When different gases were successively introduced into the chamber at a fixed temperature, dynamic changes were observed (Fig. 3). ${ }^{(8)}$ A PL emission peak was blueshifted in helium, but was redshifted in air or nitrogen. By changing the gas atmosphere, the peak are shifted back and forth reversibly.

These phenomena are interpreted in terms of molecule adsorption/desorption. The pressure change also induces a similar transition. The transition behavior of PL peaks was quantitatively examined using a pressure-controlled chamber. ${ }^{(15)}$ The ethanol gas pressure dependences of the PL emission peak from the same suspended $(9,8)$ SWNT measured at various temperatures are shown in Fig. 4. The emission peak wavelength shows similar ethanol gas pressure dependences at these four temperatures, and transition pressure increases with increasing temperature. Below the transition pressures, emission

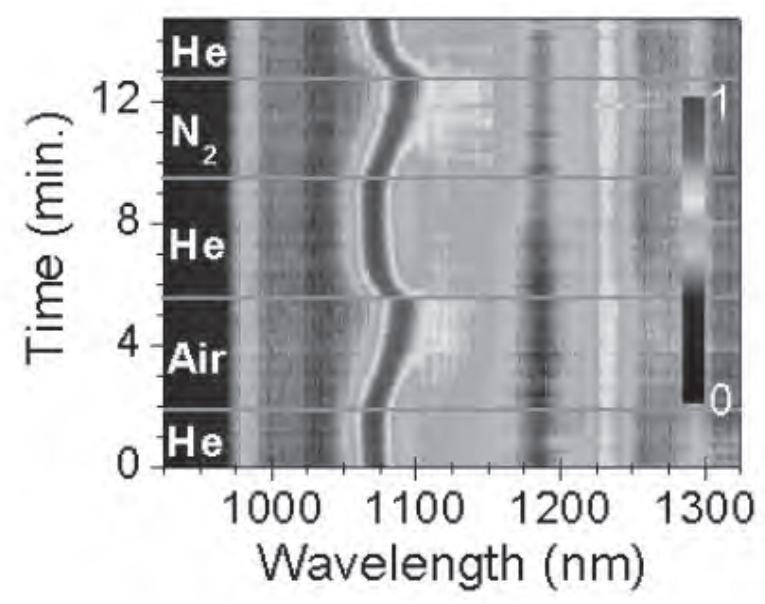

Fig. 3. Photoluminescence dynamics after the introduction of various gases. Temperature was fixed at $37^{\circ} \mathrm{C} .{ }^{(8)}$ 


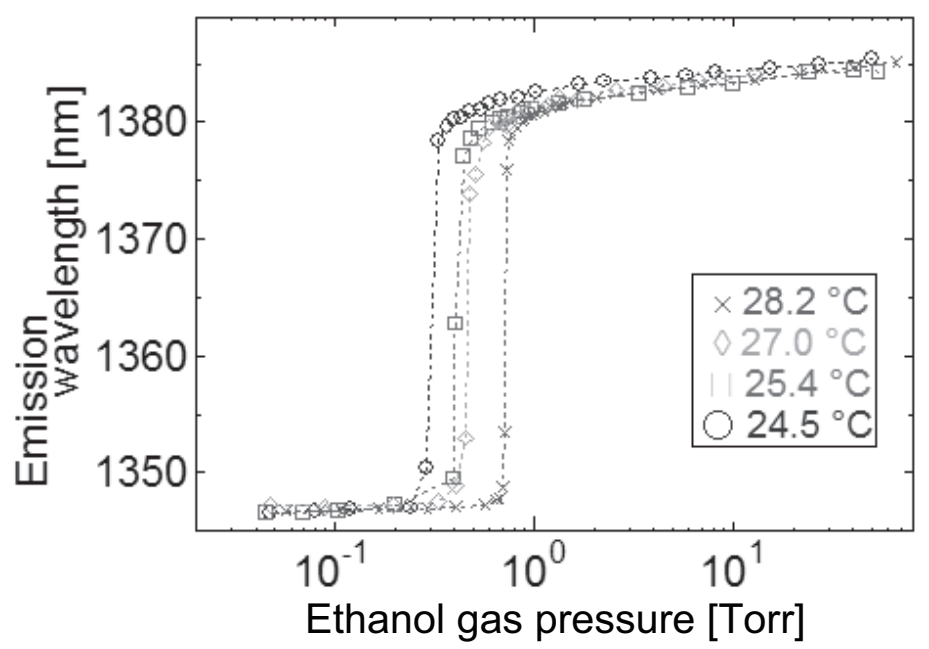

Fig. 4. Ethanol gas pressure dependences of emission wavelength at various temperatures for a (9, 8) SWNT. ${ }^{(15)}$

peak energy remains constant regardless of ethanol gas pressure and temperature. On the other hand, above the transition pressures, emission peak wavelength slightly increases with increasing ethanol pressure. The ethanol pressure dependence of PL peak energy with the rapid energy shift is quantitatively described by the adsorption and desorption of ethanol molecules on the SWNT surface based on the modified Langmuir model. ${ }^{(16)}$ In this model, the interactions among adsorbents are considered as well as those between an adsorbent and the surface. The adsorption rate $v_{+}$of gas molecules on the surface is expressed in the standard form

$$
v_{+}=\frac{P}{\sqrt{2 m k_{\mathrm{B}} T}}(1-\theta)
$$

where $P, m$, and $\theta$ are the pressure, mass, and surface coverage of gas molecules, respectively. For the desorption rate $v_{-}$, the interaction between adsorbents is taken into account:

$$
v_{-}=v_{0} \exp \left(-\frac{E+z w \theta}{k T}\right) \theta,
$$

where $v_{0}$ is a proportional constant, $E$ is the desorption energy at zero coverage, $z$ is the number of neighboring molecules, and $w$ is the lateral interaction energy between adjoining adsorbents. The tube diameter dependence of desorption energy is assumed 
to be $E=E_{\mathrm{g}}-A / d$, where $d$ is the tube diameter, $A$ is a constant, and $E_{\mathrm{g}}$ is the desorption energy of ethanol on graphite at zero coverage $(29 \mathrm{~kJ} / \mathrm{mol}){ }^{(17)}$ At the critical pressure $P_{\mathrm{t}}$, which is the pressure corresponding to the critical coverage $\theta_{\mathrm{c}}=0.5$,

$$
\frac{P_{\mathrm{t}}}{\sqrt{T} P_{0}} \frac{1-\theta_{\mathrm{c}}}{\theta_{\mathrm{c}}}=\exp \left[\frac{A / d-\left(E_{\mathrm{g}}+z w \theta_{\mathrm{c}}\right)}{k T}\right],
$$

where $P_{0}=v_{0}(2 \pi m k)^{1 / 2}$. The transition pressures of four different chirality SWNTs are measured at various temperatures from 23 to $29^{\circ} \mathrm{C}$. Figure 5 shows the relationship between $1 / T$ and transition pressure. When $A$ and $P_{0}$ are $29 \mathrm{~kJ} \mathrm{~nm} / \mathrm{mol}$ and $2.2 \times 10^{6}$ Torr $/ K^{1 / 2}$ respectively, the experimental data agree with the fitting lines. The temperature and tube diameter dependences of the transition pressure $P_{\mathrm{t}}$ are thus described in terms of the curvature effect of desorption energy.

In air ambient, suspended SWNTs show rapid energy shifts when sample temperature changes. ${ }^{(8)}$ From experiments using water vapor, we have confirmed that this transition is due to water molecule absorption onto or desorption from the suspended SWNTs in air. Therefore, the emission and excitation energies measured in air correspond to those of water-molecule-wrapped SWNTs. The emission and excitation energies measured below the transition pressures are the intrinsic optical transition energies of SWNTs. Figure 6 shows the emission and excitation wavelengths, which were measured in air ${ }^{(4,18)}$

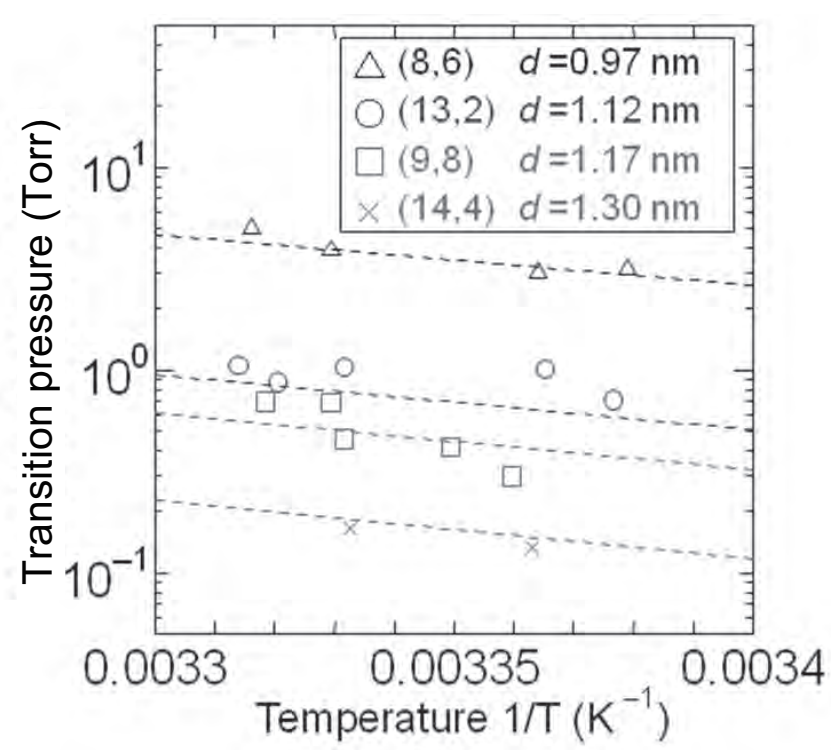

Fig. 5. Temperature and diameter dependences of the transition pressures of four chirality SWNTs. The dashed line is a fitting line based on eq. (3)..$^{(15)}$ 


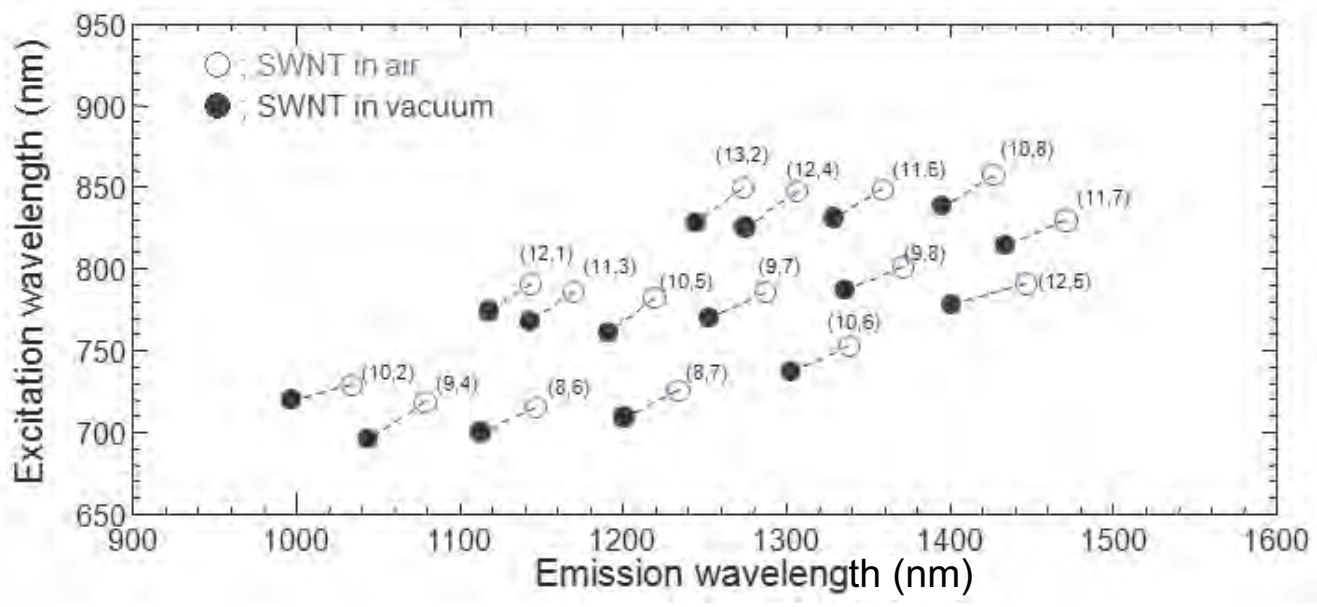

Fig. 6. Emission and excitation energies of suspended SWNTs in air (open circles) and vacuum (filled circles). ${ }^{(15)}$

and vacuum (below the transition pressure). All the PL peaks of suspended SWNTs measured in vacuum blue-shift relative to those measured in air. The average emission and excitation energy shifts are approximately 25 and $47 \mathrm{meV}$, respectively.

The dynamic transition observed by changing the ambient gas at a fixed temperature shown in Fig. 3 is also explained by the water molecule desorption. Because water molecules existed on the chamber wall, water adsorption occurred even when dry gases were introduced. Since adsorption rate is inversely proportional to the square root of molecular mass (eq. (1)), helium has a higher adsorption rate than water, air, and nitrogen at a fixed temperature and pressure. This results in a smaller water molecule coverage in helium than in air or nitrogen. Thus, the transition temperature in helium is lower than that in air or nitrogen.

For SDS-wrapped SWNTs, the energy difference between air-suspended and SDSwrapped SWNTs depends on the chiral angle and type of SWNT, which is explained by uniaxial strain generated by the surrounding SDS. For gas adsorption, no such chirality dependence was observed. ${ }^{(8,15)}$ Therefore, the type-dependent optical transition energy is specific to wrapping by large molecules.

\section{Conclusions}

Suspended SWNTs emit intense photoluminescence, reflecting the electronic excitation and emission between 1D singular peaks. Optical transitions are strongly affected by the surrounding environment, even by the adsorption of molecules. Photoluminescence intensity shows a rapid transition with the adsorption or desorption 
of condensed molecules on the nanotube surface. The transition shows a clear diameter dependence, reflecting the effect of curvature on desorption energy. Thus, the adsorption/ desorption dynamics on the nanosurface can be probed using photoluminescence. These features of SWNTs may be useful for sensing applications.

\section{Acknowledgements}

The authors acknowledge the support for their research in the form of a Grant-in-Aid for Scientific Research on Priority Areas (No.19054015) from the Ministry of Education, Culture, Sports, Science and Technology (MEXT), Japan.

\section{References}

1 O. Brand, G. K. Fedder, J. G. Korvink and O. Tabata: Carbon Nanotube Devices: Properties, Modeling, Integration and Applications (Wiley-VCH, Weinheim, 2008).

2 M. J. O'Connell, S. M. Bachilo, C. B. Huffman, V. C. Moore, M. S. Strano, E. H. Haroz, K. L. Rialon, P. J. Boul, W. H. Noon, C. Kittrell, J. Ma, R. H. Hauge, R. B. Weisman and R. E. Smalley: Science 297 (2002) 593.

3 J. Lefebvre, Y. Homma and P. Finnie: Phys. Rev. Lett. 90 (2003) 217401.

4 J. Lefebvre, J. M. Fraser, Y. Homma and P. Finnie: Appl. Phys. A 78 (2004) 1107.

5 T. Ando: J. Phys. Soc. Jpn. 66 (1997) 1066.

6 Y. Ohno, S. Iwasaki, Y. Murakami, S. Kishimoto, S. Maruyama and T. Mizutani: Phys. Status Solidi B 244 (2007) 4002.

7 Y. Miyauchia, R. Saito, K. Sato, Y. Ohno, S. Iwasaki, T. Mizutani, J. Jiang and S. Maruyama: Chem. Phys. Lett. 442 (2007) 394.

8 P. Finnie, Y. Homma and J. Lefebvre: Phys. Rev. Lett. 94 (2005) 247401.

9 Y. Homma, S. Chiashi and Y. Kobayashi: Rep. Prog. Phys. 72 (2009) 066502.

10 S. M. Bachilo, M. S. Strano, C. Kittrell, R. H. Hauge, R. E. Smalley and R. B. Weisman: Science 298 (2002) 2361.

11 R. B. Weisman and S. M. Bachilo: Nano Lett. 3 (2003) 1235.

12 J. Lefebvre, J. M. Fraser, P. Finnie and Y. Homma: Phys. Rev. B 69 (2004) 075403.

13 L. X. Benedict, S. G. Louie and M. L. Cohen: Phys. Rev. B 52 (1995) 8541.

14 J. Lefebvre, S. Maruyama and P. Finnie: Carbon Nanotubes, Advanced Topics in the Synthesis, Structure, Properties and Applications, eds. A. Jorio, M. S. Dresselhaus and G. Dresselhaus (Springer, Berlin, 2008) p. 287.

15 S. Chiashi, S. Watanabe, T. Hanashima and Y. Homma: Nano Lett. 8 (2008) 3097.

16 A. W. Adamson: Physical Chemistry of Surfaces (4th ed.) (John Wiley \& Sons, New York, 1982).

17 P. L. Walker: Chemistry and Physics of Carbon (Marcel Dekker, New York, 1970).

18 Y. Ohno, S. Iwasaki, Y. Murakami, S. Kishimoto, S. Maruyama and T. Mizutani: Phys. Rev. B 73 (2006) 235427. 